\title{
Mellin Transform Based Performance Analysis of FFH $M$-ary FSK Using Product Combining Against Partial Band Noise Jamming
}

\author{
Sohail Ahmed, Lie-Liang Yang and Lajos Hanzo \\ School of ECS, University of Southampton, SO17 1BJ, UK. \\ Tel: +44-23-8059 3364, Fax: +44-23-8059 4508 \\ Email: \{sa03r,lly,lh\}@ecs.soton.ac.uk; http://www-mobile.ecs.soton.ac.uk
}

\begin{abstract}
We propose a novel bit error ratio (BER) analysis technique for fast frequency hopping (FFH) assisted $M$-ary frequency shift keying (MFSK) using product combining (PC), when the channel is contaminated by partial band noise jamming (PBNJ). Exploiting the fact that the Mellin transform of the product of independent random variables is the product of their Mellin transforms, we derive the probability density function (PDF) of the PC's output, when communicating in uncorrelated Rayleigh fading channels contaminated by PBNJ. Derivation of the PDF thus allows computation of the system's BER. It is shown that the Mellin transform substantially simplifies the analysis of the $P C$ receiver and facilitates for the first time the analysis of FFH-MFSK PC receiver for modulation orders of $M>2$.
\end{abstract}

\section{INTRODUCTION}

Fast frequency hopping (FFH) $M$-ary frequency shift keying (MFSK) using product combining (PC) has been shown to efficiently combat partial band noise jamming (PBNJ) [1][3]. In [1]-[4], the authors have analyzed a PC aided FFH binary frequency shift keying (BFSK) receiver under various fading and jamming conditions. However, these analysis techniques have been applied to BFSK and are unsuitable for a modulation order of $M>2$. Motivated by the fact that the Mellin transform may be employed for deriving the probability density function (PDF) of a product of random variables [5][7], we opted for employing this powerful technique for analyzing PC aided FFH-MFSK. The Mellin transform of a random variable having a PDF of $f(x)$ is given by [6], [7]

$$
\mathcal{M}[f(x), z]=\int_{0}^{\infty} x^{z-1} f(x) d x,
$$

while the corresponding inverse Mellin transform returns the $\mathrm{PDF}$ and is defined as [6], [7]

$$
f(x)=\int_{c-i \infty}^{c+i \infty} x^{-z} \mathcal{M}[f(x), z] d z,
$$

where we have $i=\sqrt{-1}$ and the integration is carried out along any line parallel to the imaginary axis and lying within the strip of analyticity [6].

The novel contribution of this paper is that the proposed Mellin transform based technique substantially simplifies the

The financial support of Higher Education Commission, Pakistan, the EPSRC, UK and that of EU under the auspices of the Phoenix and Newcom projects is gratefully acknowledged. bit error ratio (BER) analysis of FFH aided $M$-ary FSK using $P C$, when the transmitted signal is corrupted by PBNJ and Rayleigh fading, and hence facilitates for the first time its analysis for $M>2$. In our previous contribution on this topic [8], we considered the same problem, although no jamming of the transmitted signal was assumed, whereas in this contribution the channel is assumed to be contaminated by PBNJ. Furthermore, in [8] we used square-law detection [9] of the FFH-MFSK signal. By contrast, in this contribution we employ envelope detection [9] and we will demonstrate in Section IV that this results in more convenient computation of the BER for a wide range of system parameters. Furthermore, we will show in Section II that for envelope detection, the random variable at the PC's output is a product of Rayleigh variables, while in [8], it was characterized by a product of Gamma variables. In [10], the PDF of a product of Rayleigh distributed variables has been derived by employing the Mellin transform. However, the inverse of the Mellin transform has been derived using Meijer's G-function in [10], whereas we will employ the residues method for this purpose [5], [8], owing to its conceptual simplicity.

The rest of this paper is structured as follows. In Section II, the relevant statistics as well as the corresponding BER expression are derived using the proposed technique. In Section III our numerical results are discussed and, finally, in Section IV, our conclusions are presented.

\section{BER ANALYSIS}

The system studied is similar to that considered in [2] and [4], except that we have a modulation order of $M \geq 2$. In the FFH-MFSK transmitter the MFSK signal modulates the carrier generated by a frequency synthesizer, which is controlled by the $L$-tuple FFH address generated by a pseudonoise (PN) generator, where $L$ is the number of frequency hops per symbol. The hop interval $T_{h}$ is related to the symbol interval $T_{s}$ by $T_{h}=T_{s} / L$. The separation between adjacent frequency tones is assumed to be $R_{h}=1 / T_{h}$, which also represents the bandwidth occupied by a single FFH-MFSK tone.

The channel is assumed to be a frequency-flat Rayleigh fading medium for each of the transmitted frequencies. Furthermore, we assume that the separation between the adjacent 


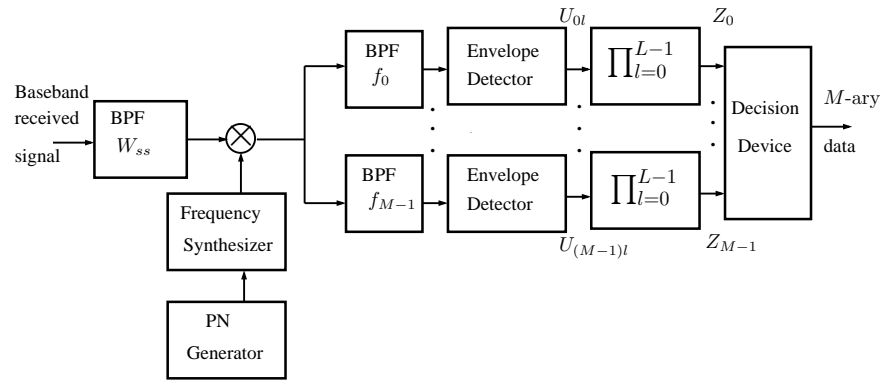

Fig. 1. Receiver schematic of the FFH-MFSK receiver using PC

frequencies is higher than the coherence bandwidth of the channel. Therefore, all FFH tones conveying the same symbol experience independent fading.

The transmitted signal is also corrupted by additive white Gaussian noise (AWGN) and a PBNJ signal having singlesided power spectral densities of $N_{0}$ and $N_{J}$, respectively. We assume that the PBNJ signal jams a fraction $0 \leq \rho \leq 1$ of the total spread spectrum bandwidth $W_{s s}$. We also assume that the PBNJ signal is contiguous and hence all $M$ FSK tones of a particular band are jammed, if the jamming signal is present in that band. Thus, the probability that a band or a tone is jammed is given by $\rho$, while the probability that the band is not jammed is $(1-\rho)$.

The receiver schematic is shown in Fig. 1, where a bandpass filter (BPF) removes any frequencies that fall outside the bandwidth $W_{s s}$. Then the frequency de-hopper of Fig. 1, which is identical to and aligned with the frequency hopper of the transmitter, de-spreads the recieved signal by exploiting the knowledge of the transmitter's unique FFH address. The demodulator is comprised of $M$ branches each corresponding to a single MFSK tone and consisting of an envelope detector [9] as well as a PC. At the output of the PC one of the $M$ decision variables is obtained.

When the signal suffers from Rayleigh fading, the output of the noncoherent envelope detector is also Rayleigh distributed [9]. Therefore, assuming that the first of the $M$ tones is activated by the transmitter, it can be shown that the PDF of the envelope detector's output signal $U_{m l}, m=$ $0,1, \ldots, M-1, l=0,1, \ldots, L-1$, corresponding to the desired signal tone, when the transmitted signal is jammed by PBNJ is given by [4], [9]

$$
\begin{gathered}
f_{U_{0 l}}^{(J)}(x)=\frac{x}{2\left(\sigma_{0}^{2}+\sigma_{J}^{2}\right)\left(1+\gamma_{h t}\right)} \\
\times \exp \left(\frac{-x^{2}}{2\left[\sigma_{0}^{2}+\sigma_{J}^{2}\right]\left[1+\gamma_{h t}\right]}\right), \quad x \geq 0,
\end{gathered}
$$

where $\gamma_{h t}=b E_{b} /\left(N_{T} L\right)$ is the signal to interference plus noise ratio (SINR) per hop, $N_{T}=N_{0}+N_{J} / \rho, E_{b}$ is the transmitted energy per bit and $b$ is the number of bits per symbol. Furthermore, in (3) the superscript $(J)$ explicitly indicates that the tone suffers from PBNJ and $\sigma_{0}^{2}=R_{h} N_{0}$ and $\sigma_{J}^{2}=R_{h} N_{J} / \rho$ represent the variance of the AWGN and the PBNJ, respectively.

By contrast, when the signal is not jammed, the correspond- ing PDF can be expressed as [4], [9]

$$
f_{U_{0 l}}(x)=\frac{x}{2 \sigma_{0}^{2}\left(1+\gamma_{h}\right)} \exp \left(\frac{-x^{2}}{2 \sigma_{0}^{2}\left[1+\gamma_{h}\right]}\right), \quad x \geq 0,
$$

where $\gamma_{h}=b E_{b} /\left(N_{0} L\right)$ is the signal to noise ratio (SNR) per hop. Similarly, the corresponding expressions for the PDFs of the undesired tones of $m=1,2, \ldots, M-1$, are given by [4], [9]

$$
f_{U_{m l}}^{(J)}(x)=\frac{x}{2\left(\sigma_{0}^{2}+\sigma_{J}^{2}\right)} \exp \left(\frac{-x^{2}}{2\left[\sigma_{0}^{2}+\sigma_{J}^{2}\right]}\right), \quad x \geq 0, m>0
$$

and

$$
f_{U_{m l}}(x)=\frac{x}{2 \sigma_{0}^{2}} \exp \left(\frac{-x}{2 \sigma_{0}^{2}}\right), \quad x \geq 0, m>0 .
$$

Therefore, the Mellin transforms of the PDFs given by (3), (4), (5) and (6) may be expressed using (1) as [6], [8], [11]

$$
\begin{gathered}
\mathcal{M}\left[f_{U_{0 l}^{(J)}}(x), z\right]=\alpha_{J}^{(1-z) / 2} \Gamma(z / 2+1 / 2), \\
\mathcal{M}\left[f_{U_{0 l}}(x), z\right]=\alpha^{(1-z) / 2} \Gamma(z / 2+1 / 2), \\
\mathcal{M}\left[f_{U_{m l}^{(J)}}(x), z\right]=\beta_{J}^{(1-z) / 2} \Gamma(z / 2+1 / 2), \quad m>0
\end{gathered}
$$

and

$$
\mathcal{M}\left[f_{U_{m l}}(x), z\right]=\beta^{(1-z) / 2} \Gamma(z / 2+1 / 2), \quad m>0,
$$

where we have $\alpha_{J}=1 /\left[2\left(\sigma_{0}^{2}+\sigma_{J}^{2}\right)\left(1+\gamma_{h t}\right)\right], \alpha=1 /\left[2 \sigma_{0}^{2}(1+\right.$ $\left.\left.\gamma_{h}\right)\right], \beta_{J}=1 /\left[2\left(\sigma_{0}^{2}+\sigma_{J}^{2}\right)\right]$ and $\beta=1 / 2 \sigma_{0}^{2}$.

Assuming that $p$ out of the $L$ hops of a symbol are jammed, the output of the $m$ th PC, $m=0,1, \ldots, M-1$, seen in Fig. 1 may be expressed as

$$
Z_{m}=\prod_{l=0}^{p-1} U_{m l}^{(J)} \prod_{l=0}^{L-p-1} U_{m l}
$$

We will now exploit the fact that the Mellin transform of the product of independent random variables is equal to the product of the Mellin transforms of the individual random variables [6], [12] for the sake of reducing the numerical computational complexity involved. Hence, the Mellin transform of the $m$ th PC's output, given that $p$ out of the $L$ hops are jammed, may be expressed as

$$
\begin{aligned}
\mathcal{M}\left[f_{Z_{m}}\left(y_{m} \mid p\right), z\right] & =\left[\mathcal{M}\left[f_{U_{m l}}^{(J)}(x), z\right]\right]^{p} \\
& \times\left[\mathcal{M}\left[f_{U_{m l}}(x), z\right]\right]^{L-p},
\end{aligned}
$$

where $f_{Z_{m}}\left(y_{m} \mid p\right)$ represents the PDF of the $m$ th PC output, $m=0,1, \ldots, M-1$. Consequently, from (7) and (8) for the desired signal tone, we have

$$
\begin{aligned}
\mathcal{M}\left[f_{Z_{0}}\left(y_{0} \mid p\right), z\right] & =\alpha_{J}^{p(1-z) / 2} \alpha^{(L-p)(1-z) / 2} \\
& \times \Gamma^{L}(z / 2+1 / 2),
\end{aligned}
$$

while from (9) and (10) for the undesired (nonsignal) tones, corresponding to $m>0$, we have

$$
\begin{aligned}
\mathcal{M}\left[f_{Z_{m}}\left(y_{m} \mid p\right), z\right] & =\beta_{J}^{p(1-z) / 2} \beta^{(L-p)(1-z) / 2} \\
& \times \Gamma^{L}(z / 2+1 / 2) .
\end{aligned}
$$


Then, using (2), the PDF of $Z_{0}$ can be generated as the inverse Mellin transform of (13), yielding

$$
\begin{aligned}
f_{Z_{0}}\left(y_{0} \mid p\right) & =\int_{c-i \infty}^{c+i \infty} y_{0}^{-z} \alpha_{J}^{p(1-z) / 2} \alpha^{(L-p)(1-z) / 2} \\
& \times \Gamma^{L}[(z+1) / 2] d z .
\end{aligned}
$$

We now invoke the change of variable technique and replace $(z+1) / 2$ by $z$ in the above equation, since this does not affect the path of integration [6]. Hence, after further simplification, we have

$f_{Z_{0}}\left(y_{0} \mid p\right)=2 \int_{c-i \infty}^{c+i \infty} y_{0}^{1-2 z} \alpha_{J}^{p(1-z)} \alpha^{(L-p)(1-z)} \Gamma^{L}(z) d z$.

Upon applying the residue theorem [5]-[8], [13] to (16), we have

$$
\begin{aligned}
f_{Z_{0}}\left(y_{0} \mid p\right) & =\sum_{j} \operatorname{Res}\left[2 y_{0}^{1-2 z} \alpha_{J}^{p(1-z)}\right. \\
& \left.\times \alpha^{(L-p)(1-z)} \Gamma^{L}(z)\right]_{(z=-j)},
\end{aligned}
$$

where $\operatorname{Res}[\cdot]_{(z=-j)}$ represents the residue at the $j$ th pole of the integrand. The PDF of $Z_{0}$ can be determined upon numerically evaluating (17) by using symbolic mathematics based software, such as Maple or Mathematica. However, in what follows we also derive analytical expressions for the PDFs $f_{Z_{0}}\left(y_{0} \mid p\right)$ and $f_{Z_{m}}\left(y_{m} \mid p\right)$, with the aid of [5], [6], [8], [13], [14].

It is widely recognized that the function $\Gamma(z)$ has an infinite number of poles at $z=-j$ for $j=1,2, \ldots$, and at each of these poles the corresponding residue is given by [13], [14]

$$
\left.\Gamma(z)(z+j)\right|_{(z=-j)}=\frac{(-1)^{j}}{j !} .
$$

Since $\Gamma^{L}(z)$ in (17) has an $L$ th-order pole at each integer value of $z=-j$, with the aid of [5], [6], [13], [14], we have

$$
\begin{aligned}
f_{Z_{0}}\left(y_{0} \mid p\right) & =\frac{2 \alpha_{J}^{p} \alpha^{L-p} y_{0}}{(L-1) !} \sum_{j=0}^{\infty} \frac{d^{L-1}}{d z^{L-1}} \\
& \times\left[\left(\alpha_{J}^{p} \alpha^{L-p} y_{0}^{2}\right)^{-z} \Gamma^{L}(z)(z+j)^{L}\right]_{(z=-j)} .
\end{aligned}
$$

Letting $\mathcal{U}(z)=\left(\alpha_{J}^{p} \alpha^{L-p} y_{0}^{2}\right)^{-z}$ and $\mathcal{V}(z)=\Gamma^{L}(z)(z+j)^{L}$, we have [8], [14]

$$
\begin{aligned}
f_{Z_{0}}\left(y_{0} \mid p\right) & =\frac{2 \alpha_{J}^{p} \alpha^{L-p} y_{0}}{(L-1) !} \sum_{j=0}^{\infty} \sum_{r=0}^{L-1}\left(\begin{array}{c}
L-1 \\
r
\end{array}\right) \\
& \times\left[\mathcal{U}^{(r)}(z) \mathcal{V}^{(L-1-r)}(z)\right]_{(z=-j)},
\end{aligned}
$$

where $\mathcal{U}^{(r)}(z)$ and $\mathcal{V}^{(r)}(z)$ denote the $r$ th derivatives of $\mathcal{U}(z)$ and $\mathcal{V}(z)$, respectively. Then, it can be readily shown that we have

$$
\left.\mathcal{U}(z)\right|_{(z=-j)}=\left(\alpha_{J}^{p} \alpha^{L-p} y_{0}^{2}\right)^{j}
$$

and

$$
\left.\mathcal{U}^{(r)}(z)\right|_{(z=-j)}=\left[-\ln \left(\alpha_{J}^{p} \alpha^{L-p} y_{0}^{2}\right)\right]^{r}\left(\alpha_{J}^{p} \alpha^{L-p} y_{0}^{2}\right)^{j},
$$

while, following a number of manipulations with the aid of [5], [6], [8], [13], [14], we arrive at

$$
\left.\mathcal{V}(z)\right|_{(z=-j)}=\frac{(-1)^{j L}}{(j !)^{L}} .
$$

and

$$
\begin{aligned}
&\left.\mathcal{V}^{(r)}(z)\right|_{(z=-j)}=L\left\{\left.\sum_{t=0}^{r-1}\left(\begin{array}{c}
r-1 \\
t
\end{array}\right) \mathcal{V}^{(t)}(z)\right|_{(z=-j)}\right. \\
& \times \quad(-1)^{r-t}(r-1-t) ! \sum_{k=0}^{\infty}\left[\frac{1}{(1+k)^{r-t}}\right] \\
&\left.+\left.\quad \sum_{t=0}^{r-1}\left(\begin{array}{c}
r-1 \\
t
\end{array}\right) \mathcal{V}^{(t)}(z)\right|_{(z=-j)} \sum_{k=1}^{j} \frac{1}{(k)^{r-t}}\right\}
\end{aligned}
$$

The interested reader may refer to [8] for the complete derivation of the above equation. Hence, the PDF of $Z_{0}$, conditioned on the assumtpion that $p$ out of the $L$ hops are jammed, can be determined from (20), while the PDF of $Z_{m}$, $m>0$ can be similarly expressed as [5], [6], [8], [13], [14]

$$
\begin{aligned}
f_{Z_{m}}\left(y_{m} \mid p\right) & =\frac{2 \beta_{J}^{p} \beta^{L-p} y_{m}}{(L-1) !} \sum_{j=0}^{\infty} \sum_{r=0}^{L-1}\left(\begin{array}{c}
L-1 \\
r
\end{array}\right) \\
& \times\left[\mathcal{U}_{m}^{(r)}(z) \mathcal{V}^{(L-1-r)}(z)\right]_{(z=-j)},
\end{aligned}
$$

where

$$
\left.\mathcal{U}_{m}(z)\right|_{(z=-j)}=\left(\beta_{J}^{p} \beta^{L-p} y_{m}^{2}\right)^{j}
$$

and

$$
\left.\mathcal{U}_{m}^{(r)}(z)\right|_{(z=-j)}=\left[-\ln \left(\beta_{J}^{p} \alpha^{L-p} y_{m}^{2}\right)\right]^{r}\left(\beta_{J}^{p} \beta^{L-p} y_{m}^{2}\right)^{j} .
$$

Thus, the symbol error ratio (SER), conditioned on the assumption that $p$ out of the $L$ hops are jammed by PBNJ, is given by [9]

$$
P_{s}(p)=1-\int_{0}^{\infty} f_{Z_{0}}\left(y_{0} \mid p\right)\left[\int_{0}^{y_{0}} f_{Z_{m}}\left(y_{m} \mid p\right) d y_{m}\right]^{M-1} d y_{0}
$$

The overall average SER is therefore given by [1]

$$
P_{s}=\sum_{p=0}^{L}\left(\begin{array}{l}
L \\
p
\end{array}\right) \rho^{p}(1-\rho)^{L-p} P_{s}(p) .
$$

Given the SER, the corresponding BER can be determined using the relation of $P_{b}=\frac{M / 2}{M-1} P_{s}$ [9].

\section{NUMERICAL RESULTS AND DISCUSSION}

In this section, we validate our analysis detailed in Section II using numerical results and discuss the achievable performance of the system employing the proposed method. The integrations seen in (28) are performed numerically. It has been found based on our results not presented here owing to lack of space that using a limited number of residues, i.e $j \leq 30$, is sufficient for computing both the PDF and the BER accurately.

Using (20), we portray the PDF of the PC's output corresponding to the FFH-MFSK signal tone in Fig. 2, assuming $M=4, L=3, E_{b} / N_{0}=10 d B$ in the absence of jamming. 
The PDF of the PC's output assuming square-law detection has also been shown using results from [8]. It can be seen in Fig. 2 that beyond a certain value pf PC's output, envelope detection of the received signal leads to smaller area under the tail of its PDF compared to square-law detection. The high probability of encountering high PC output values for squarelaw detection are due to its squaring operation. By contrast, as observed in Fig. 2, the PDF has a more limited dynamic range, when envelope detection is employed. Since accurate numerical integration in (28) requires the truncation of the $\mathrm{PDF}$ at a point where it becomes negligible, the employment of envelope detection facilitates the numerical computation of the BER.

Using (28), we portray our BER results for various values of the modulation order in Fig. 3, where the parameter values of $L=3, \rho=0.1$ and $E_{b} / N_{0}=20 d B$ have been assumed. Fig. 3 demonstrates that our analytical results match the simulation results. The results of this figure also show that the performance of the system improves, as the modulation order is increased. This result is in agreement with our prior knowledge in the context of $M$-ary systems [9].

In Fig. 4, the effects of increasing the diversity order on the system BER has been characterised. In this figure, the parameters of $M=8, \rho=0.1$ and $E_{b} / N_{0}=20 \mathrm{~dB}$ have been assumed. Fig. 4 demonstrates that increasing the value of $L$ enhances the achievable diversity gain, which results in an improved BER. We also note in Fig. 4 that at $L=5$, the analytical results slightly deviate from the simulation results, especially when $E_{b} / N_{J}$ approaches $20 d B$. The reason for this inaccuracy is that having a high diversity order leads to a high expected PC output and consequently increased area under the tail of its PDF, which prevents the accurate computation of (20), (25) and (28). Another reason for the inaccuracy of these results is that the computation of the SER at high values of $L$ incurrs higher-order differentials, which result in roundoff errors involved in the computation of (20) and (25).

In Fig. 5, we have plotted the BER for $M=32, L=3$, $E_{b} / N_{0}=20 d B$ and for various values of the PBNJ duty factor $\rho$. Similar performance results have been plotted in Fig. 6 for the parameter values of $M=16, L=4$ and $E_{b} / N_{0}=20 \mathrm{~dB}$. We note from these figures that the BER performance of the system using $M$-ary FSK follows similar trends to those reported in the context of BFSK in [4]. Specifically, as seen in Figs. 5 and $6, \rho=1$ results in the worst BER performance, and hence for $\rho<1$ a better BER performance is recorded. This observation demonstrates that PBNJ associated with a duty factor of unity constitutes the worst-case jamming scenario in Rayleigh fading channels. This result is in agreement with previous results on FFH-BFSK PC systems designed for combatting PBNJ [1]-[4]. However, as seen in Figs. 5 and 6, as the signal power increases in comparison to the jammer power, the performance of the system becomes less sensitive to $\rho$.

We also note in Figs. 5 and 6 that the analytical results pertaining to $\rho=0.01$ slightly deviate from the corresponding simulation results. The reason for this difference is that at a low PBNJ duty factor, the effective jammer power encountered in a jammed hop increases, contributing to the increased magnitude of the received signal. Thus, in this scenario the resultant $\mathrm{PC}$ output may become high and the area under the tail of its PDF may increase. Consequently, under these conditions, the computation of the PDFs and the BER using (20), (25) and (28) may be less accurate. Note that the difference in the analytical and simulation results is more significant in Fig. 6, where higher value of $L$ has been used as compared to Fig. 5. This is because higher diversity order also contributes towards inaccuracy in computation of (28), as explained above.

It has been observed in our investigations not shown here for reasons of space economy that the computation of the BER becomes challenging for $L>5$, for low values of the jamming duty factor as well as for $E_{b} / N_{J}$ and $E_{b} / N_{0}>$ $20 d B$ in conjunction with high $M$, and hence some numerical precision may have to be sacrificed. Nonetheless, the results are fairly reliable for a wide range of system parameter values of practical interest.

\section{CONCLUSION}

We have used the Mellin transform for analyzing the BER performance of the classic FFH-MFSK PC receiver, operating in a Rayleigh fading channel contaminated by PBNJ. The PDF of the PC's output was determined in a closed form, as seen in (20) and (25). With the aid of these PDF expressions, the BER of the system was evaluated numerically. The proposed technique has been shown to be accurate and the analytical results obtained using (20), (25) and (28) match the simulation results for most practical values of the modulation order, PBNJ duty factor, for $L \leq 5$ and for $E_{b} / N_{J}$ and $E_{b} / N_{0} \leq$ $20 d B$. Our analytical results demonstrate that PBNJ having a jamming duty factor of unity results in the worst-case jamming scenario for the FFH-MFSK system communicationg over Rayleigh fading channels. Moreover, the BER performance of the system improves, as either the modulation order or the diversity order is increased. Our future research may focus on the investigation of the same system considered in this contribution, when communicating over more generalized classes of fading channels, such as Nakagmai- $m$ channels.

\section{REFERENCES}

[1] K. C. Teh, A. C. Kot, and K. H. Li, "Performance analysis of FFH/BFSK product combining receivers under multitone jamming," IEEE Transactions on Vehicular Technology, vol. 48, pp. 1946-1953, November 1999.

[2] K. C. Teh, A. C. Kot, and K. H. Li, "Partial band jamming rejection of FFH/BFSK with product combining receiver over a Rayleigh-fading channel," IEEE Cimmunications Letters, vol. 1, pp. 64-66, May 1997.

[3] T. C. Lim, W. He, and K. Li, "Rejection of partial-band noise jamming with FFH/BFSK product combining receiver over Nakagami-fading channel," IEEE Communications Letters, vol. 34, pp. 960 - 961, May 1998.

[4] G. Huo and M. S. Aluoini, "Another look at the BER performance of FFH/BFSK with product combining over partial-band jammed Rayleigh fading channels," IEEE Transactions on Vehicular Technology, vol. 50, pp. 1203-1215, September 2001.

[5] A. M. Mathai, "Products and ratios of generalized gamma variates," Skandinavisk Aktuarietidskrif, no. 55, pp. 193-198, 1972.

[6] M. D. Springer, The algebra of random variables. New York: John Wiley and sons, 1979.

[7] I. H. Sneddon, The use of integral transforms. New York: Mcgraw-Hill Book Company Inc., 1972.

[8] S. Ahmed, L. L. Yang, and L. Hanzo, "Mellin Transform Based Performance Analysis of Fast Frequency Hopping Using Product Combining," in Proceedings of the IEEE Vehicular Technology Conference, 2006., vol. 4, pp. 1635- 1639, May 2006. 
[9] J. G. Proakis, Digital communications. Singapore: Mcgraw-Hill, 2001.

[10] J. Salo, H. M. El-Sallabi, and P. Vainikainen, "The distribution of the product of independent Rayleigh random variables," IEEE Transactions on Antennas and Propagation, vol. 54, pp. 639 - 643, February 2006.

[11] H. Bateman, Tables of integral transforms, Volume I. New York: Mcgraw-Hill Book Company Inc., 1954.

[12] P. Galambos and I. Simonelli, Products of Random Variables: Applications to problems of physics and to arithmatical functions. New York: Marcel Dekker Inc., 2004.

[13] S. Lang, Complex analysis. New York: Springer-Verlag New York, Inc., 1999.

[14] I. Gradshteyn and I. M. Ryzhik, Tables of integrals, series and products. London: Academic Press, 1965.

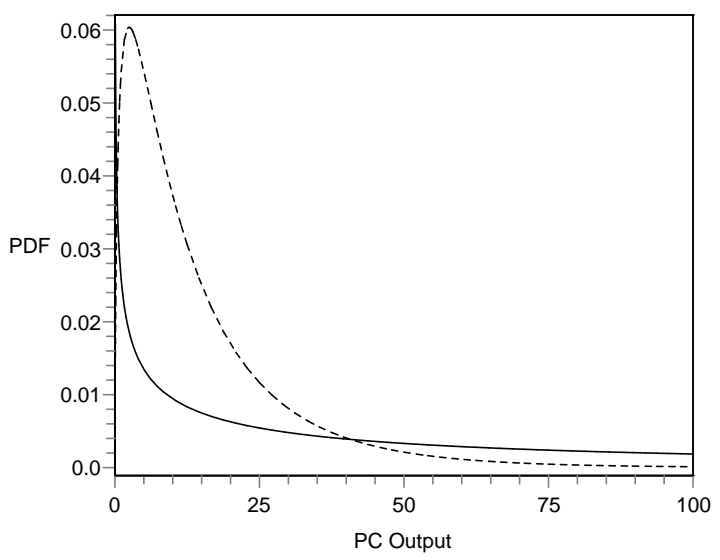

Square-law detection
Envelope detection

Fig. 2. The PDF of PC output for both square-law and envelope detection of desired tone, for a FFH aided 4-ary FSK PC receiver, communicating over a Rayleigh fading channel, assuming $L=3$ and $E_{b} / N_{0}=10 \mathrm{~dB}$.

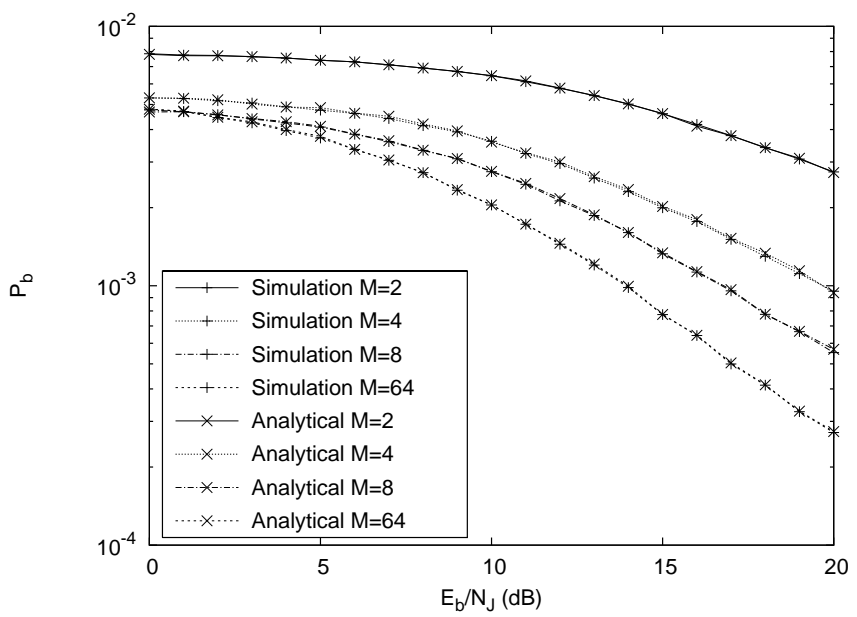

Fig. 3. Comparison of the analytical results of (28) and the simulation results of the BER versus the $E_{b} / N_{J}$ performance of a FFH aided MFSK PC receiver communicating over a Rayleigh fading channel, assuming $L=3$, $E_{b} / N_{0}=20 d B, \rho=0.1$ and various $M$ values.

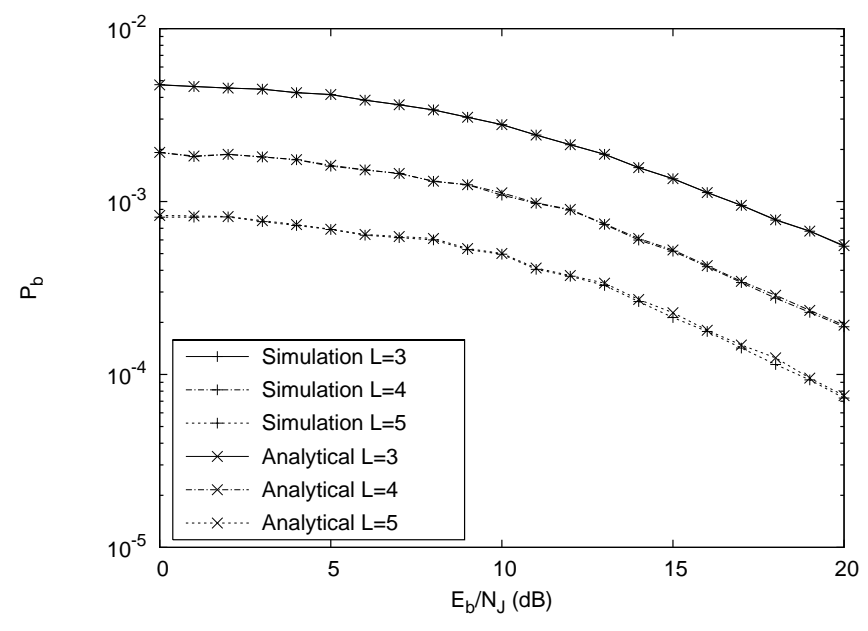

Fig. 4. Comparison of the analytical results of (28) and the simulation results of the BER versus the $E_{b} / N_{J}$ performance of a FFH aided 8-ary FSK PC receiver communicating over a Rayleigh fading channel, assuming $E_{b} / N_{0}=20 d B, \rho=0.1$ and various $L$ values.

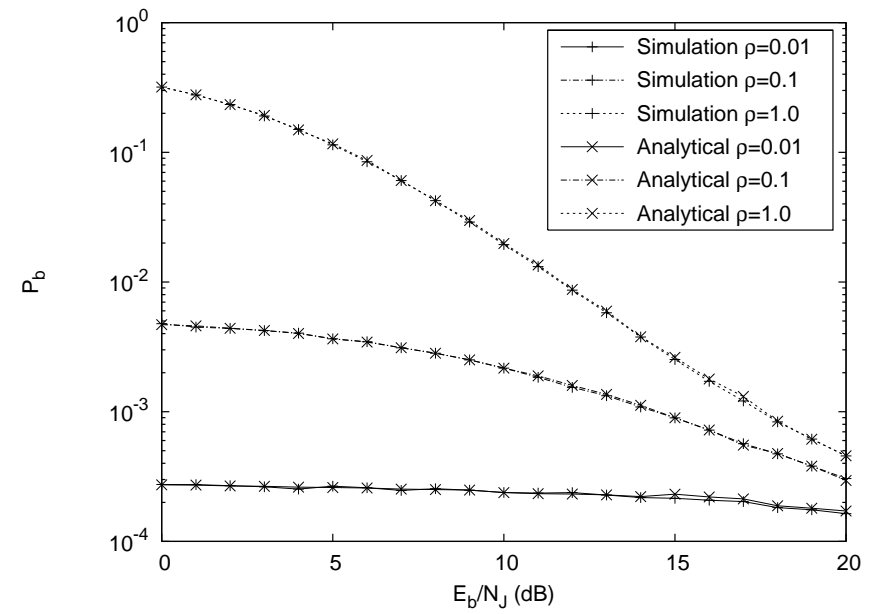

Fig. 5. Comparison of the analytical results of (28) and the simulation results of the BER versus the $E_{b} / N_{J}$ performance of a FFH aided 32-ary FSK PC receiver communicating over a Rayleigh fading channel, assuming $L=3$, $E_{b} / N_{0}=20 d B$ and various values of the PBNJ duty factor $\rho$.

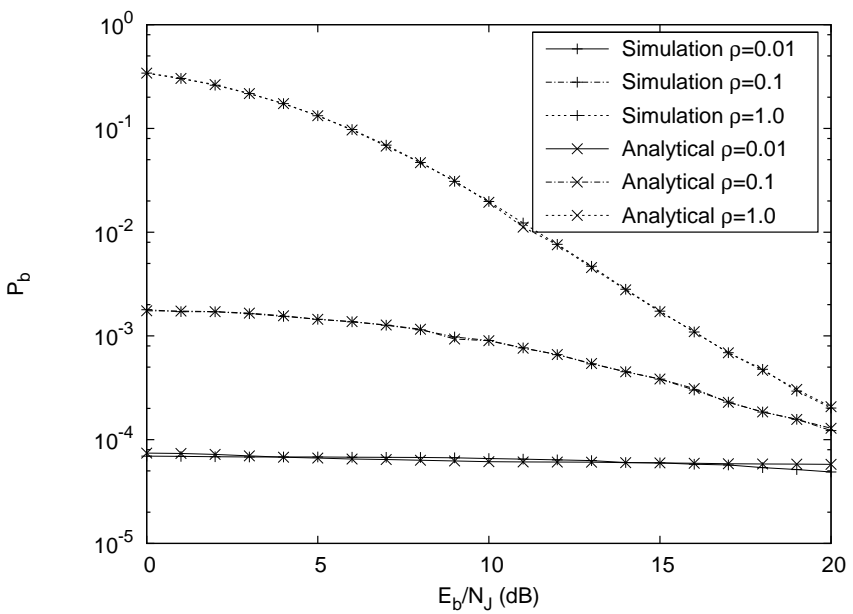

Fig. 6. Comparison of the analytical results of (28) and the simulation results of the BER versus the $E_{b} / N_{J}$ performance of a FFH aided 16-ary FSK PC receiver communicating over a Rayleigh fading channel, assuming $L=4$, $E_{b} / N_{0}=20 d B$ and various values of the PBNJ duty factor $\rho$. 